\title{
Synthetical element evaluation method for AC-DC EHV converter substation power quality
}

\author{
Yuan Ye ${ }^{1, a}$, Zhu Daming 2,b \\ ${ }^{1}$ State Grid Jilin Electric Power Research Institute, Renmin Street NO.4433,Changchun 130021, \\ China \\ ${ }^{2}$ State Grid Jilin Electric Power Company, Renmin Street NO.4629, Changchun 130021, China \\ a zenkiyy163@163.com, ${ }^{\mathrm{b}}$ zxt@yeah.com,
}

Keywords: AC-DC EHV Converter Substation; Power Quality; Harmonic Monitoring Platform.

Abstract. Nowadays, with the development of the EHV grid and area of megacity, such as Shanghai, Nanjing. There are many factories and AC-DC converter substations are involved into the urban region, which causes much more power quality problems than before, so that more and more people focused on the power supply using experience. In this article, we analyzed several evaluation methods of power quality used for now, then we make in-depth study about the characteristics in power supply for urban region and AC-DC EHV converter substations. And with the construction work of harmonic monitoring platform of State Grid, we can make a real-time judgment for power supply points, such as government, hospital, bank and so on. At last, we put forward a new power quality evaluation method for AC-DC converter substations. The method is based on the unique power quality parameters of power supply in city, it is established from four elements, which are: feature harmonic voltage, feature harmonic current, feature power and voltage change, all these elements constitute a method to evaluate the power quality of AC-DC EHV converter substations. And in the final result can be showed in a single symbol, which includes all the four elements.

\section{Introduction}

For a long time, people always focus on the power quality of new energy power supplies, factories, smelteries and so on. But there are not many people care about the power quality about their own family, especially the area nearby the AC-DC converter substations.

In the past, the electric equipment is simple and easy, such as lamps, electrical motors, and so on, so people do not care about the power quality for themselves. But with the development of domestic electric equipment, more and more precise instrument are used in family grid, for example, high-class rice cooker, high power variable frequency air conditioner, and so on, all these electrical equipments are much more strict with power quality. And with the development of digital industry, more and more Big Data Center are built in the urban area. All these electric equipments require a much more rigorous power quality index.

Under this circumstance, the State Grid Company were building a harmonics monitoring platform for these years, which covers most area of the grid. That made us can estimate the status by the monitoring devices in real-time. But when we were evaluating power quality for a single point, we can not tell the users the power quality of one point is qualified or not directly ${ }^{[3]}$, due to the power quality involved so many indicators, and each region of the grid structure, the power supply, load types each are not identical, we can only tell users which indicator is qualified or unqualified. So that we need a handy method to estimate power quality for a single point or some specified points ${ }^{[1,6]}$.

This article summarize the differences between different power quality methods ${ }^{[4,5]}$, generalized the representative influences caused by AC-DC converter substations, and collected various demands for all kinds of users. At last, we present a new method called "synthetical element evaluation" to make a quick evaluation for a points nearby a AC-DC EHV converter substations, which is based on the harmonics monitoring platform. 


\section{Concerns of Power Quality for Different Consumers}

\section{A. Kinds of Consumers}

There are various kinds of users for power supply, such as domestic consumers, government sector, hospitals, banks, large shopping malls, precise instruments manufactures and so on, and all their demands in power quality are different ${ }^{[2,9]}$.

B. Focus of Diverse Consumers

The most important demand for domestic users is the stability of power supply, there are surveys shows that, the limitation of tolerance for the domestic users is 10 minutes without notifications ${ }^{[1]}$. But nowadays, there are more and more families pay attention to the power quality for their home, because more and more advanced electrical equipments are used, which are much more vital for power quality than the normal equipments.

For the Big Data centers, the most vital focus is harmonics, voltage dips, voltage swells and short interruptions, so they always equip some treatment facilities. For these users, even 100ms voltage interruption or voltage dip can cause very huge economy lost for these centers.

To the hospitals and banks, they mainly focused on the stability of power and voltage, harmonics of current and voltage. Because of the equipment they used, all the equipments are very sensitive to the power fluctuations and harmonics changes.

For the government users, the most important concerns are the reliability of power supply, so there are always several charging lines for the government.

For the precise instruments manufactures, the crucial thing is the harmonics voltage, harmonics current, and voltage unbalances. In the other hand, these users always produce some harmonics voltage and current in their manufacturing process.

\section{Harmonics Monitoring Platform}

\section{A. Structure of Harmonics Monitoring Platform}

Recent years, more and more power quality issues occurred, these issues caused lots of damage to the users ${ }^{[7,8]}$. So that the power supply company realize that power quality had already became a serious problem for both consumers and the companies themselves. In this case, the harmonics monitoring platform was established, from 2006, more than 6000 monitoring devices are installed in all voltage levels of transformer substations. And the data scale became very huge for now, this gave us a firm foundation of data to analyze and estimate the status of power grid. As is shown in fig 1 .

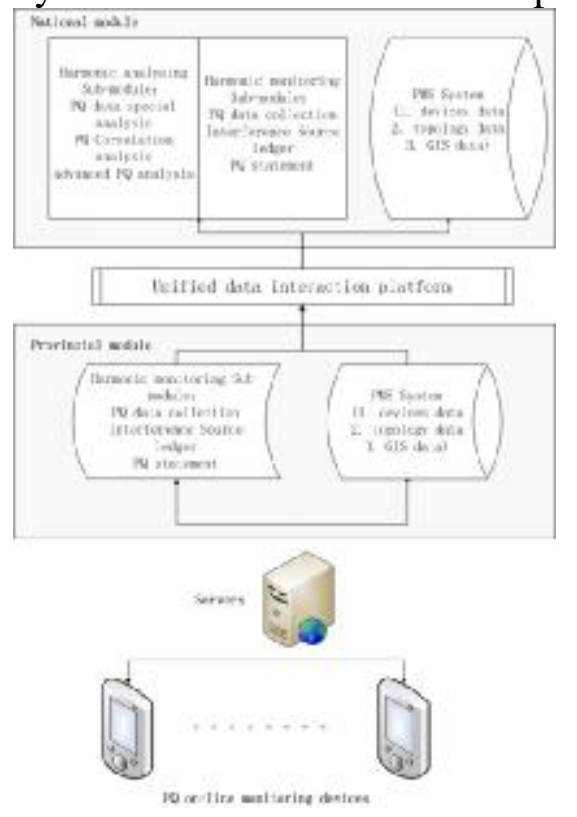

Fig 1. Structure of harmonics monitoring platform 


\section{B. Disadvantage of Harmonics Monitoring Platform}

Form the structure, we can see that the platform is mainly focused on every single index of power quality, so that we can not get a quick result for a point, we have to check every index to make sure if there is any unqualified index of power quality. This would take much time for a technician to give out the result when they were evaluating the power quality for some users.

Especially when we wanted to evaluate the power quality indexes for a period of some users, we must inspect all the indexes for the period which we want to check. And this obviously would take a very long time for the technician to finish this work. But if we can present a new indicator which can represent some vital indexes that we cared, it will reduce a lot of time to finish the evaluation.

\section{Methods of the Assessment in the System of Power Quality}

\section{A. Methods of Power Quality Assessment}

The work of power quality assessment is based on the parameters of the power grid, there are more than 10 standards for power quality, and there are always several indicators in one standard, so that there are more than 50 indicators for all the power quality. Obviously we can not make an evaluation with all index, we need to select some more important indexes to finish the assessment.

Due to the power quality index is numerous, there are many ways to do the assessment ${ }^{[7]}$, according to different application environment and the external conditions, different methods got very big distinction, rough classification of evaluation way as shown in figure 2.

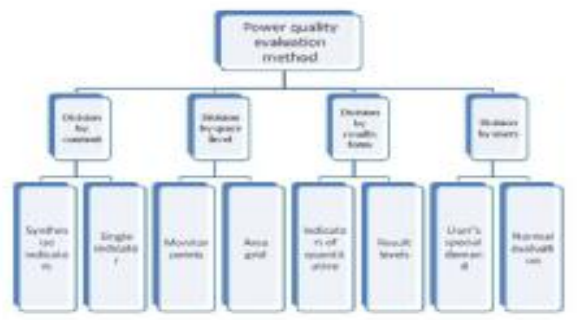

Fig 2. Classification of Power Quality Estimation Methods

As we can see from figure 2, a variety of power quality evaluation methods are put forward foe now, according to different needs assessment object, methods also have very big difference. Therefore, we need to distinguish these methods in different areas, and adjust measures to local conditions to develop a suitable for this type of power quality system evaluation method ${ }^{[10]}$.

B. Emphases of Power Quality Estimation

The evaluation of the single index is a way for one index of power quality. It is always carried out to evaluate a specific index of power quality for particular aim $^{[3]}$. The integration evaluation is based on the single evaluation, which integrates some of the similar indexes, and then gets a new quantitative evaluation result.

Quantitative evaluation and grade assessment are the methods used to evaluate different demands for application environments. The index quantification assessment is to compare the evaluation result of the index to the standard or the value in the contract between users and power supply company ${ }^{[4]}$, so that we can get a more intuitive result. The grade assessment is based on the special requirements, always from consumers, and the index of the power quality are divided into different evaluation levels by the rules from users.

The methods of monitoring point evaluation and regional assessment are divided according to the area, which is to evaluate the difference between a point or an area. It is easy to understand, mainly focus on particular purpose.

User's special demand and normal evaluation is mainly used for the users and power supply company. This two kind of methods are always carried out for some particular transformer substation, especially by users' demand.

In this paper, we make a use of aforesaid method, which is based on the results of evaluation index of content, form three methods to the needs of users of the evaluation process, focus on urban region 
accounts for the larger area of power quality system evaluation method has carried on the induction and summary ${ }^{[1]}$.

\section{Synthetical Elements Evaluation Method}

We present a synthetical element evaluation method, which is based on the harmonics monitoring platform of State Grid.

A. Significant Index of AC-DC EHV converter substations region

According to the users, we consider the process of power quality assessment for AC-DC EHV converter substations area should be mainly focused on the following aspects.

(1) Harmonic voltage, in the AC-DC converter substations area, because of the diverse of power application, various electric equipments make influence to the others, especially the great use of inverter air-conditioner;

(2) Harmonic current, the same as the harmonic voltage, there is much harmonic current when the equipment make influence to the others, sometimes the voltage is more apparent but sometimes the current is much more apparent;

(3) Power, for the Big Data center, banks and hospitals, these consumer are very sensitive to the power factor and power fluctuation, especially the stability of power;

(4) Stability of voltage, this element is very signification for precise manufactures, elevators in large shopping malls and Big Data center.

B. Synthetical Element Evaluation Method Composition

According to the characteristics of AC-DC converter substations area, we put forward a synthetical element evaluation method. First we choose 12 evaluation indexes for this method, and detailed indicators are shown in table I. then we conclude these 12 indexes into four more significant items that needed to be paid attention to, as is shown in fig 3.

Table I. Indexes to calculate

\begin{tabular}{|c|c|c|c|c|c|}
\hline 1 & Typical harmonic voltage & 2 & $\begin{array}{c}\text { Total harmonic voltage } \\
\text { distortion }\end{array}$ & 3 & Long time flickers \\
\hline 4 & Typical harmonic current & 5 & $\begin{array}{c}\text { Total harmonic current } \\
\text { distortion }\end{array}$ & 6 & $\begin{array}{c}\text { Negative sequence } \\
\text { current }\end{array}$ \\
\hline 7 & Power factor & 8 & Stability of power & 9 & Voltage deviation \\
\hline 10 & $\begin{array}{c}\text { Voltage dip and short } \\
\text { interruptions }\end{array}$ & 11 & Voltage swells & 12 & $\begin{array}{c}\text { Negative sequence } \\
\text { voltage }\end{array}$ \\
\hline
\end{tabular}

In this evaluation method, we induce these 12 indexes into four elements. And the classification method for these indicators is shown in figure 3.

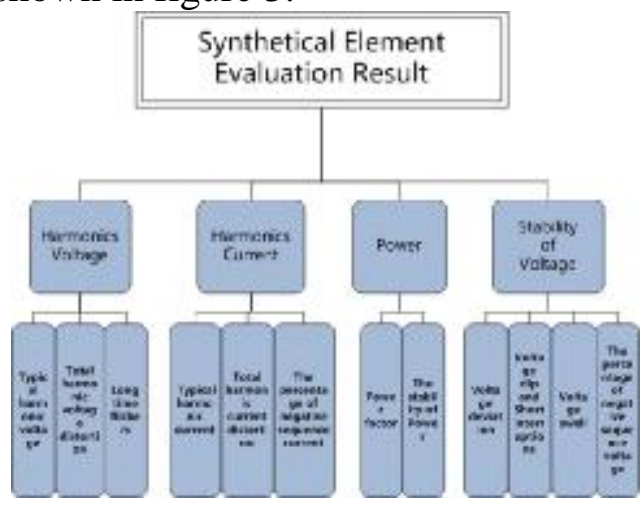

Fig 3. Synthetical Evaluation Composition

C. How to calculate in this method

After obtaining the value of the above 12 indexes, here we set it as " $v$ ", then we compare these numbers with the standard values, here we set the standard values as "s", then we can get a preliminary outcome. And the ways to calculate scores are shown in table II. 
Table II. Score of Calculation Formula

\begin{tabular}{|c|c|}
\hline Values & Point \\
\hline$(\mathrm{v} / \mathrm{s})<0.6$ & 3 \\
\hline $0.6 \leq(\mathrm{v} / \mathrm{s})<0.9$ & 2 \\
\hline $0.9 \leq(\mathrm{v} / \mathrm{s})<1$ & 1 \\
\hline$(\mathrm{v} / \mathrm{s}) \geqslant 1$ & 0 \\
\hline
\end{tabular}

For example, there is a $220 \mathrm{kV}$ substation 5 time harmonic voltage is $1.2 \%$, according to the national standard in the limit of 5 time harmonic voltage of $220 \mathrm{kV}$ is $1.6 \%$, v/s $=1.2 / 1.6=0.75$, according to the methods in table 2, then the assessment score is 2 points for this $220 \mathrm{kV}$ substation 5 times harmonic voltage.

After we get all the scores of the indexes, we can make the final evaluation of this point. Here we set every element's final score as " $r$ ", and we set all the full marks as " $t$ ". Then we can get a final evaluation result, the meaning of results is shown in table III.

Table III. Evaluation Result

\begin{tabular}{|c|c|}
\hline Value & Meaning \\
\hline$(\mathrm{r} / \mathrm{t})<0.6$ & Failed \\
\hline $0.6 \leq(\mathrm{r} / \mathrm{t})<0.75$ & Qualified \\
\hline $0.75 \leq(\mathrm{r} / \mathrm{t})<0.9$ & Good \\
\hline$(\mathrm{r} / \mathrm{t}) \geqslant 0.9$ & Excellent \\
\hline
\end{tabular}

After we complete calculating of monomer level determination of four components, according to the characteristics of the evaluation project and the surrounding grid environment, the scores of the four elements of assessment level of weight distribution, here we set four patterns to allot the weightiness for every element. As it is shown in fig 4.

\begin{tabular}{|c|c|c|c|c|}
\hline Weightiness & Pattern1 & Pattern2 & Pattern3 & Pattern4 \\
\hline W1 & 0.3 & 0.25 & 0.2 & 0.2 \\
\hline W2 & 0.3 & 0.25 & 0.2 & 0.3 \\
\hline W3 & 0.2 & 0.25 & 0.3 & 0.25 \\
\hline W4 & 0.2 & 0.25 & 0.3 & 0.25 \\
\hline
\end{tabular}

Fig 4. Weightiness distribution

At last, we concluded those four elements into a final evaluation results, as shown in figure 5. And the formula for final result is the same as shown in table III.

And if technicians prefer to use figures to show the result, we can also show the result in figures, there is nothing different in the formula to calculate.

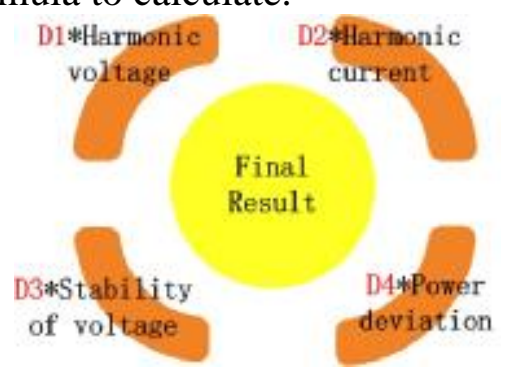

Fig 5. Finally Evaluation Results

\section{Application of Synthetically Element Evaluation}

We take an application for $220 \mathrm{kV}$ substation for example in this article, which is in the AC-DC converter substations area and there are several factories supplied by AC-DC converter substations to this substation, so that the harmonic voltage and harmonic current is much more important than the others. In this example, we choose pattern 1 to do the math. 


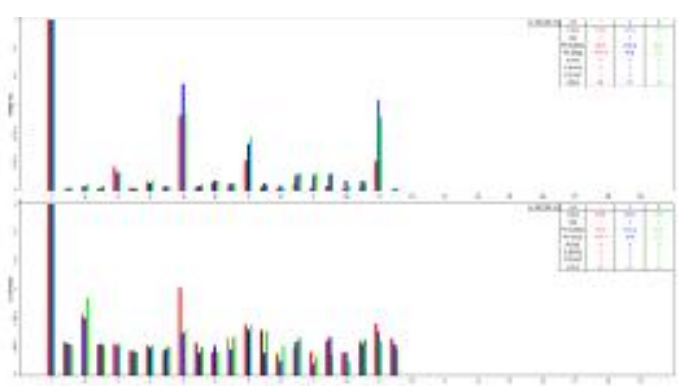

Fig 6. Harmonic spectrum analysis of substation

As can be seen from the figure 6, the typical harmonic voltage of the substation is 5,7 and 11 times. But the harmonic current contents are different at all, the typical harmonic current of the substation is 2 and 5 times. The figures are shown in table IV, here we got 15 indicator, so the full score is $45(3 * 15=45)$.

Table IV. Harmonic Voltage of Substation

\begin{tabular}{|c|c|c|c|c|c|}
\hline \multicolumn{2}{|c|}{ PARAMETER } & Phase A & Phase B & Phase C & Limit \\
\hline \multirow{2}{*}{$\begin{array}{c}\text { Typical } \\
\text { Harmonic } \\
\text { voltage }(\%)\end{array}$} & 5 & 1.04 & 1.54 & 1.85 & 1.6 \\
\cline { 2 - 6 } & 7 & 0.73 & 0.96 & 1.21 & 1.6 \\
\hline \multicolumn{2}{|c|}{ THDu(\%) } & 0.36 & 1.08 & 0.87 & 1.6 \\
\hline \multicolumn{2}{|c|}{ Flicker } & 1.13 & 1.87 & 1.55 & 2 \\
\hline
\end{tabular}

Table V. Harmonic Voltage Result

\begin{tabular}{|c|c|c|c|c|}
\hline \multirow{2}{*}{ ELEMENT } & INDEX & \multicolumn{2}{|c|}{ SCORE } & RESULT \\
\hline \multirow{2}{*}{$\begin{array}{c}\text { Harmonic } \\
\text { Voltage }\end{array}$} & Typical Harmonic Voltage & 15 & & \multirow{2}{*}{29} \\
\cline { 2 - 3 } & THDu & 6 & 0.65 \\
\cline { 2 - 3 } & Long time Flicker & 8 & & \\
\hline
\end{tabular}

As we can see from table 5, the element of harmonic voltage score is 29 , the full score for the element is 45 , so that the result for this element is 0.65 , means qualified.

Table VI. Four Components Scores

\begin{tabular}{|c|c|c|c|c|}
\hline Element & Point & Weightiness & Result & Final Result \\
\hline Harmonic voltage & 0.65 & 0.3 & 0.195 & \\
\hline Harmonic current & 0.97 & 0.3 & 0.291 & 0.8 \\
\hline Power & 0.76 & 0.2 & 0.152 & (Good) \\
\hline Stability of voltage & 0.81 & 0.2 & 0.162 & \\
\hline
\end{tabular}

According to the formula in table 2 and table 3, we can get results for all the four elements, and so that the final results, as we can see in table 6 and fig 7 .

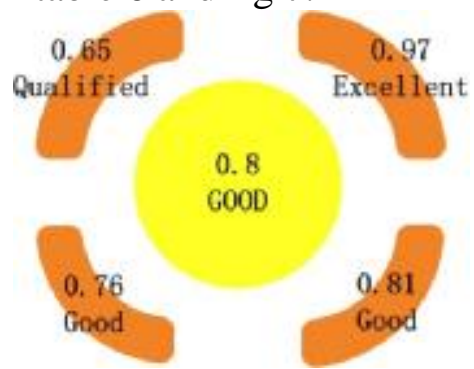

Fig 7. Finally Evaluates the Results 


\section{Conclusion}

In this article, we put forward a "synthetical element evaluation" evaluation method to assess power quality of area around a AC-DC converter substations. The method classified 12 indexes into four elements. Then we assign different weightiness for every elements based on the grid structure and the composition of power source in this area. And we also present a formula to calculate every indicator and every element, which is easily to understand and implement. At last, we evaluate a $220 \mathrm{kV}$ substation by "synthetical element evaluation" method, as shown in table 6 and fig 7, we can easily tell the advantage and disadvantage of power quality, this shows that the "synthetical element evaluation" method is simple and feasible to be used in the harmonics monitoring platform.

\section{References}

[1] Zhou Hui, Yang Honggeng, Wu Chuanlai. A power quality comprehensive evaluation method based on grey clustering. Power System Protection and Control, 2012,40(15):70-75.

[2] Zhou Lin, Li Qiu-hua, Liu Hua-yong, Zhang Feng,Qu Yongjie. Evaluation of Power Quality by Fuzzy Artificial Neural Network. HIGH VOLTAGE ENGINEERING, 2007, 33(9):66-69.

[3] Yang Zhixin , Yuan Xiaodong, Bai Jingjing. Synthetic evaluation of power quality based on improved TOPSIS and RSR method. Power Demand Side Management, 2013, (5):11-16.

[4] Chen Haitao, Data analysis and analysis of power quality monitoring [D]. Guang Zhou: South China University of Technology, 2013.

[5] Wang Fang, Gu Wei, Yuan Xiaodong. Power quality management platform for smart grid. Electric Power Automation Equipment, 2012, 32(7): 134-139.

[6] Fu Xueqian, Chen Haoyong. Comprehensive power quality evaluation based on weighted rank sum ration method. Electric Power Automation Equipment, 2015, 35(1): 128-132.

[7] Huang Yongwei, Liu Jun, Zhou Xichao. Simulation Analysis of Power Quality Characteristics of Wind Farm and Control Measures. Industry and Mine Automation,2012,8(8):67-70.

[8] Bai Hong-bin, Wang Rui-hong. Influence of the Grid-connected Wind Farm on Power Quality. Proceedings of the Chinese Society of Universities, 2012,24(1):120-124.

[9] Li Guodong, Li Gengyin, Yang Xiaodong, Zhou Ming. A Comprehensive Power Quality Evaluation Model Based on Radar Chart Method. AUTOMATION OF ELECTRIC POWER SYSTEMS, 2010, 34(14):70-74.

[10] Li Ruqi, Su Haoyi. A Synthetic Power Quality Evaluation Model Based on Extension Cloud Theory. Automation of Electric Power Systems, 2012, 36(1):66-70.

Ye Yuan (male 1984-), received his master degree in 2011from Beijing Jiao Tong University, then he joined State Grid Jilin Electric Power Research Institute, mainly engaged in the work of power quality status evaluation and power quality testing. 